\title{
Understanding and Predicting Trends in Urban Freight Transport
}

\author{
Petar Mrazovic ${ }^{1}$, Bahaeddin Eravci ${ }^{2}$, Josep L. Larriba-Pey ${ }^{3}$, Hakan Ferhatosmanoglu ${ }^{4}$, Mihhail Matskin ${ }^{5}$ \\ ${ }^{1,5}$ Dept. of Software and Computer Systems, Royal Institute of Technology, Stockholm, Sweden \\ 2,4 Dept. of Computer Engineering, Bilkent University, Ankara, Turkey \\ 1,3 Dept. of Computer Architecture, Polytechnic University of Catalonia, Barcelona, Spain \\ ${ }^{4}$ Max Planck Institute for Informatics, Saarbruecken, Germany \\ Email: ${ }^{1}$ mrazovic@kth.se, ${ }^{2}$ beravci@gmail.com, ${ }^{3}$ larri@ac.upc.edu, ${ }^{4}$ hakan@cs.bilkent.edu.tr, ${ }^{5}$ misha@kth.se
}

\begin{abstract}
Among different components of urban mobility, urban freight transport is usually considered as the least sustainable. Limited traffic infrastructures and increasing demands in dense urban regions lead to frequent delivery runs with smaller freight vehicles. This increases the traffic in urban areas and has negative impacts upon the quality of life in urban populations. Data driven optimizations are essential to better utilize existing urban transport infrastructures and to reduce the negative effects of freight deliveries for the cities. However, there is limited work and data driven research on urban delivery areas and freight transportation networks. In this paper, we collect and analyse data on urban freight deliveries and parking areas towards an optimized urban freight transportation system. Using a new check-in based mobile parking system for freight vehicles, we aim to understand and optimize freight distribution processes. We explore the relationship between areas' availability patterns and underlying traffic behaviour in order to understand the trends in urban freight transport. By applying the detected patterns we predict the availabilities of loading/unloading areas, and thus open up new possibilities for delivery route planning and better managing of freight transport infrastructures.
\end{abstract}

Index Terms - urban freight transport, smart mobility, smart cities, parking availability prediction

\section{INTRODUCTION}

Today, more than a half of the worlds population lives in cities and urban areas. This number is expected to rise to 5 billion by 2030 [1] which puts intense pressure on city resources and infrastructures. High population densities in urban environments are responsible for increasing consumption of goods and services that often need to be delivered from the less crowded city periphery. However, the traffic infrastructures and the possibilities for their extension in dense urban environments are limited, and thus deliveries tend to be made in smaller loads and frequent trips which additionally increases the traffic in already crowded urban grids of streets [2]. This also results in generating noise and pollutant emissions, increasing congestion and posing a threat to the safety of road users [3]. The social and economic concentration of resources in cities poses a strong need for efficient and sustainable freight distribution processes. In this context, mobile data management and analytics arise as a means to achieve more efficient freight distribution systems, without the need for large investments or sophisticated technologies. In this paper, we are taking the first step towards such a solution by analysing and predicting usage of urban freight transport infrastructure over data collected by a new mobile application developed for this purpose.

The provision of on-street and off-street loading/unloading areas designated for the freight vehicles has been recognized as the most effective infrastructure measure for optimising lastmile delivery operations and improving mobility conditions in urban areas [3]. Nevertheless, with the increasing urban population, urban transport demands are becoming difficult to absorb due to limitations of the urban environments. The loading ${ }^{1}$ areas are becoming over-occupied and can not meet high transport demands, and allocating new spaces for logistics activities is not possible in already dense urban environments. Therefore, the pervasive information and communication technologies naturally arise as a means to improve circulation of the freight vehicles at the loading areas.

This paper analyzes data from a new mobile crowdsourcing system that allows deliverers to check-in at the designated loading area, and thus provide real-time occupancy information. The mobile application has been released as a part of Barcelona's sustainable urban mobility plan. We focus on exploratory analysis of the check-in dataset collected from the system in order to detect temporal and spatio-temporal patterns in urban freight transport. Discovered patterns allow us to detect hot spots in the city, as well as morning and evening rush hours for individual loading areas. By applying the detected patterns we develop several availability prediction models for the loading areas in the city. In particular, we propose linear regression (LR) based models to incorporate both the historical delivery trends for the specific area and the current availability patterns for that specific day to better estimate the current availability. Our LR-based forecasting models can successfully predict available parking spaces in next 15-30 minutes. Our work naturally opens up new possibilities for delivery route planning and better managing of parking spaces and freight transport infrastructure.

The contributions of this work are summarized as follows:

\footnotetext{
${ }^{1}$ We use "loading" in short while referring to both loading and unloading activities.
} 
- We model trends in urban freight transport by analysing temporal availability of loading areas.

- We explore the relationships between an area's availability patterns and underlying traffic behaviour and locations.

- We collected a novel dataset via a new mobile checkin application, that reflects the usage of urban freight transport infrastructure for the city of Barcelona. We present exploratory statistics that give insights about the use of loading areas by delivery vehicles.

- We propose a methodology for predicting the number of unoccupied parking spaces at loading areas. We propose clustering of similar areas to overcome the problem of sparse data and harmonization of online and historical data enhancing the previous state-of-art.

- We present extensive experimental evaluation over Barcelona data that confirms the efficiency and accuracy of the proposed approach.

The rest of this paper is organized as follows. In the next section, we give a description of the urban freight transport network in the city of Barcelona. In Section III, we describe the details and some statistics about the collected data. In Section IV, we show how to estimate the real-life availability of loading areas. Afterwards, in Section V we compute areas' historical availability profiles and explore obtained temporal and spatio-temporal patterns. In Section VI, we apply the findings and propose new models for availability prediction. In Section VII we report the experimental evaluation. In Section VIII we give a brief overview of the related work. Finally, we conclude our work in Section IX.

\section{BARCELONA'S URBAN FREIGHT TRANSPORT SCHEME}

Freight requires dedicated urban spaces, such as loading areas. Insufficient delivery spaces transfer delivery activities on traffic lanes and pavements, which leads to congestions and traffic accidents. Therefore, the city of Barcelona, as well as many other European cities, has adopted land use planning and infrastructure measures for achieving a more efficient integration of urban freight in the urban transport system. In 2015 Barcelona city authorities launched a new scheme for urban freight transport which prescribes the obligatory usage of newly regulated areas for delivery operations, locally known as areaDUM (Distribuci Urbana de Mercaderies in Catalan). Roughly 9,000 parking spaces across 2,000 areas have been designated for the freight transport purposes, while an estimated 45,000 parking manoeuvres take place in these zones each day. In other to improve the road network and parking capacity, different parking regulations have been introduced for each of the loading areas. For example, some of the lateral road lanes are devoted to traffic during peak hours, deliveries during off peak hours, and residential parking during the night [4]. Additionally, different parking time limits have been prescribed for the different areas. The parking enforcement unit was formed to ensure compliance of these parking regulations to facilitate availability of the loading areas for freight vehicles.

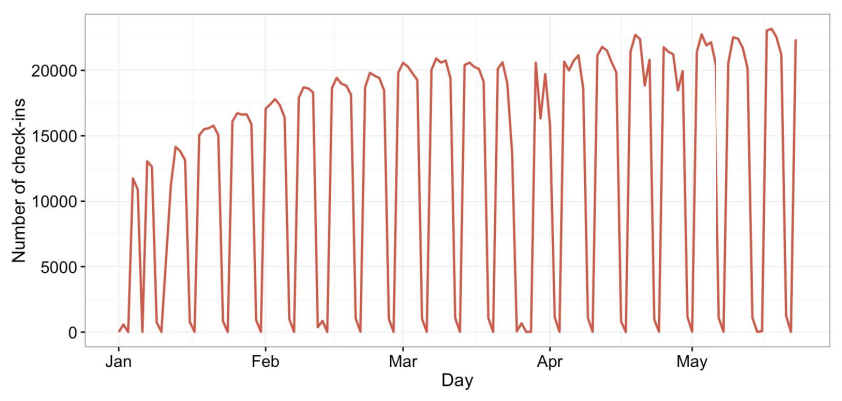

Fig. 1. Total number of check-ins per each day of the observed period between January $1^{\text {st }}, 2016$ and May $23^{\text {rd }}, 2016$

In order to facilitate and regulate the usage of loading areas, and to improve the circulation of vehicles, the city of Barcelona introduced a novel parking management solution based on mobile technologies. In order to use the designated areas, deliverers need to check-in with their vehicle's registration plate number using either mobile application or SMS service. In addition, the mobile application, available for both Android and iPhone devices, helps deliverers in managing their parking operations. The application informs deliverers about the time remaining for parking and also includes a system of warnings to help them avoid fines. The new service aims to offer more availability of spaces and, in the future, recommend the areas in which deliverers are most likely to find parking in specific time ranges.

\section{AREADUM DATASET}

In this section, we introduce the areaDUM check-in dataset, and give some basic characteristics of it. To the best of our knowledge, this is the first dataset crowdsourced from citizens and private and professional deliverers that reflects the usage of the urban freight infrastructure. The dataset consists of 49,172 users, i.e. deliverers, 1,990 loading areas and 3,014,610 checkins over the period between January $1^{\text {st }}, 2016$ and May $23^{\text {rd }}$, 2016. Each check-in consists of the ID of the deliverer who made the check-in, her vehicle's plate number (anonymised), the loading area to which the check-in was made, geographic coordinates, and a timestamp. Each of the loading areas in the dataset is geocoded, and deliverers could check in only if they were in the vicinity of the corresponding area. Therefore, the check-ins' geographic coordinates are introduced only to confirm their validity. Further, the number of authorized parking spaces for each of the loading areas is known from the dataset. There are total 8,491 parking spaces across 1,990 loading areas, while the average number of parking spaces per loading area is 4.27 .

In spite of the large amount of check-ins, the collected data is still sparse, as it is the case for most ICT based data collection and smart city applications. The new system based on mobile technologies (mobile application and SMS service) was recently introduced to replace the old parking discs. However, the two systems coexisted for a short period of time allowing users to adapt to the new system. Nevertheless, 


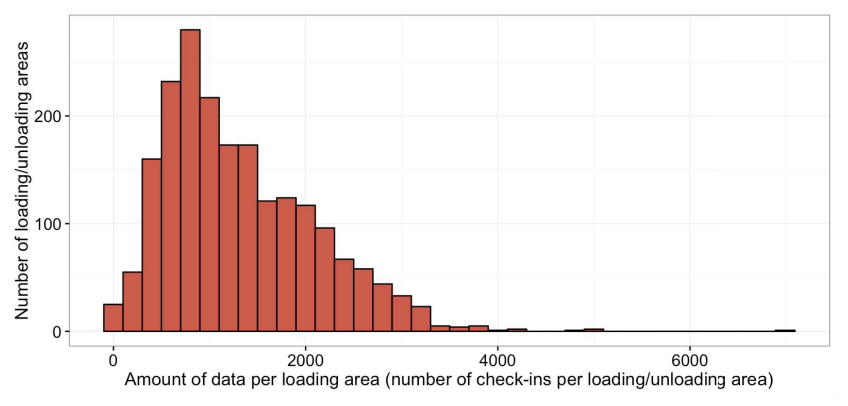

Fig. 2. Distribution of check-ins among loading areas

during the observed time period, the estimated percentage of deliverers using the new system has grown to around $60 \%$. This trend could be seen in Figure 1 where we plotted the total number of check-ins per each day of the observed period. Also notice the occasional valleys in the plot which represent holidays and weekends when the delivery operations were kept to minimum. However, in order to further address the issue of data sparsity, we have to look at the distribution of these check-ins among the loading areas. As we will see in the next section, not all of the areas have the same turnover, and thus some of them stand out with a lower number of check-ins in the dataset. Figure 2 depicts such unequal distribution of data among different loading areas. Each bar in the plot indicates the number of areas with the certain amount of check-ins (plotted on the horizontal axis). From the plot we can see there are almost 200 areas with less than 500 check-ins $(\sim 3.49$ check-ins per day). Usage of these areas is harder to predict due to sparseness of collected information, and thus one needs to put an extra effort to deal with the data sparsity.

\section{PARKing AVAilability estimation}

The new service aims to eliminate congestions at loading areas and balance the total amount of traffic in urban freight network. The first step towards achieving these goals is understanding how the parking availability changes over time and designing a prediction algorithm which can help deliverers in planning their delivery routes. Therefore, we study how to compute and predict real-life availabilities of loading areas.

We start by formally defining the availability of loading area as the total number of unoccupied parking spaces.

Definition IV.1 (Availability of loading area). Availability of loading area $\hat{a}\left(d_{i}, t_{j}\right)$ is the total number of unoccupied parking spaces at area $d_{i}$ at time $t_{j}$. Formally,

$$
\hat{a}\left(d_{i}, t_{j}\right)=c_{d_{i}}-o\left(d_{i}, t_{j}\right)
$$

where $c_{d_{i}}$ is the capacity of area $d_{i}$ (i.e. total number of parking spaces), and $o\left(d_{i}, t_{j}\right)$ is the total number of occupied parking spaces at area $d_{i}$ at time $t_{j}$.

Therefore, if we want to compute the real-life availability of a loading area $d_{i}$, we need to know the total number of occupied spaces $o\left(d_{i}, t_{j}\right)$. However, since not all deliverers have adopted the new system based on mobile technologies,

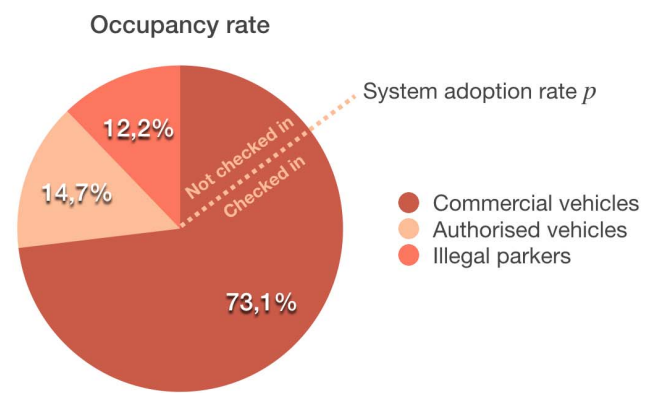

Fig. 3. Occupancy data collected in field work

this information cannot be inferred directly from the checkin dataset. Additionally, authorized emergency vehicles (e.g. police cars, fire trucks, ambulances, etc.) and vehicles with disabled parking permits can be legally parked at loading areas without checking in. Finally, the parking spaces can be also occupied by illegal parkers.

Definition IV.2 (Occupancy of loading area). Occupancy of loading area $o\left(d_{i}, t_{j}\right)$ is the total number of occupied parking spaces at area $d_{i}$ at time $t_{j}$. Formally,

$$
o\left(d_{i}, t_{j}\right)=o_{c}^{+}\left(d_{i}, t_{j}\right)+o_{c}^{-}\left(d_{i}, t_{j}\right)+o_{a}\left(d_{i}, t_{j}\right)+o_{i}\left(d_{i}, t_{j}\right)
$$

where $o_{c}^{+}\left(d_{i}, t_{j}\right), o_{c}^{-}\left(d_{i}, t_{j}\right), o_{a}\left(d_{i}, t_{j}\right)$ and $o_{i}\left(d_{i}, t_{j}\right)$ stand for the total number of parking spaces occupied by checkedin commercial vehicles, not checked in commercial vehicles, authorized vehicles and illegal parkers, respectively.

In order to estimate the real-life availability and occupancy of loading areas, Barcelona City Council conducted a field research where parking behaviours in a representative sample of loading areas have been observed. The collected data is reported in Figure 3. 73.1\% of parking spaces were occupied by commercial vehicles, $14.7 \%$ by authorized vehicles and $12.2 \%$ by illegal parkers. Additionally, notice that the ratio between checked in and not checked in commercial vehicles depends on the system's adoption rate $(p)$ which slowly increases with time (Figure 1). Therefore, when estimating and predicting real-life availability we will simulate different lifespan stages of the new system by adjusting adoption rates.

Definition IV.3 (System adoption rate). We define the system adoption rate $p\left(d_{i}, t_{j}\right)$ at loading area $d_{i}$ at time $t_{j}$ as the ratio

$$
p\left(d_{i}, t_{j}\right)=\frac{o_{c}^{+}\left(d_{i}, t_{j}\right)}{o_{c}^{+}\left(d_{i}, t_{j}\right)+o_{c}^{-}\left(d_{i}, t_{j}\right)}
$$

However, we will assume that this ratio is the same for all the $d_{i}$ and $t_{j}$. Hence, we will denote this universal quantity simply by $p$.

Using the above definitions and results from conducted field research we can estimate availability of loading areas. Since the total number of checked in commercial vehicles $o_{c}^{+}\left(d_{i}, t_{j}\right)$ is directly observable from the check-in dataset, we use it to 
express other occupancy variables. Using (3) we can express the total number of not checked in commercial vehicles as follows

$$
o_{c}^{-}\left(d_{i}, t_{j}\right)=\frac{o_{c}^{+}\left(d_{i}, t_{j}\right)(1-p)}{p}
$$

Similarly, using the statistical result reported in Figure 3 we can express the total number of parking spaces occupied by authorized and illegal vehicles as follows

$$
o_{a}\left(d_{i}, t_{j}\right)+o_{i}\left(d_{i}, t_{j}\right)=\frac{0.269}{0.731}\left(o_{c}^{+}\left(d_{i}, t_{j}\right)+o_{c}^{-}\left(d_{i}, t_{j}\right)\right)
$$

After we substitute $o_{c}^{-}\left(d_{i}, t_{j}\right)$ in (5) with the right side of (4) we get

$$
o_{a}\left(d_{i}, t_{j}\right)+o_{i}\left(d_{i}, t_{j}\right)=\frac{0.368}{p} o_{c}^{+}\left(d_{i}, t_{j}\right)
$$

Finally, by using (4) and (6) in (1) we obtain the availability estimation formula

$$
\hat{a}\left(d_{i}, t_{j}\right)=c_{d_{i}}-\frac{1.368}{p} o_{c}^{+}\left(d_{i}, t_{j}\right)
$$

In the next section we employ the derived formula in order to obtain the areas' historical availability profiles that reflect the trends in urban freight transport network.

\section{HISTORICAL AVAILABILITY PROFILES}

In this section, we study temporal delivery trends by computing individual historical availability profiles for each of the areas. The historical availability profile (HAP) of a loading area consists of the mean of the area's parking availability as a function of time. However, before computing the profiles, first we need to take a closer look at the dataset and analyse the periodicity of the underlying process in order to choose the appropriate duration of the profiles and the size of the discretisation bin.

\section{A. Seasonality analysis}

We treat the data as time series and analyse the autocorrelation functions (ACF) for each of the loading areas. Initially, we sample the time-stamped data by accumulating the number of check-ins over 1-minute sampling period. In Figure 4 we plot ACF of an example time series recovered by sampling the data from the loading area located in La Rambla, one of

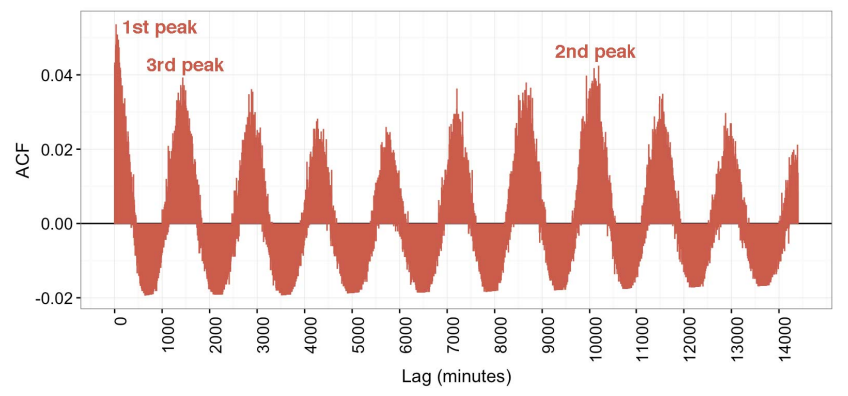

Fig. 4. ACF of the time series recovered for the example loading area located in La Rambla street

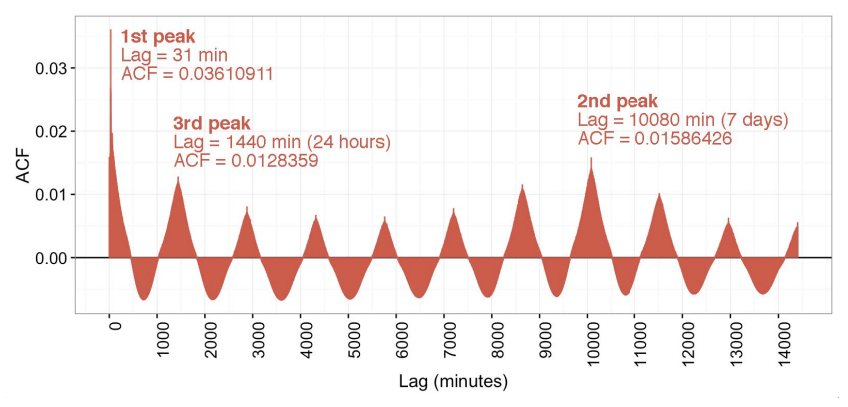

Fig. 5. Average ACF

the most popular streets in Barcelona. The plot reveals the periodicities (seasonality) of vehicle arrivals at the observed area. The AFC exhibits three significant peaks at lags of 31 , 10,188 and 1,394 minutes. The largest peak occurs at lag of 31 minutes which indicates the strongest correlation caused by the present parking regulations. More specifically, for the most of the loading areas, including the observed one, maximum allowed stay time is 30 minutes. Further, the areas are very often fully occupied and thus deliverers need to wait for a parking space. Therefore, the strong 31-minute periodicity suggest that deliverers tend to stay at the area for the maximum allowed time, whereupon the newly freed parking space is immediately taken by another vehicle. The second largest peak occurs at lag of 10,188 minutes, i.e. 7.075 days, and indicates weakly seasonality of the process. The third peak at lag of 1,394 minutes, i.e. 23.23 hours, indicates daily seasonality, while the rest of the peaks in Figure 4 represent its harmonics. Due to the seasonal nature of the urban freight demand and the established urban delivery routines, daily and weakly periodicities in the dataset have been expected a priori. For example, shops, bars and restaurants are replenished daily and weekly with stocks of fresh and dry food, and other retail goods. Finally, the discovered periodicities can be observed more clearly in Figure 5 where we average the ACFs computed for all 1,990 loading areas.

\section{B. Constructing HAPs}

For most of the loading areas, parking is permitted only during daytime, i.e. from 08:00 to 20:00. We refer to this period as operating cycle. Therefore, when constructing HAPs we compute the parking availability only for the operating cycles, assuming that for rest of the time all parking spaces are available. In other words, we split the time domain into a number of operating (daily) cycles.

Definition V.1 (Operating cycle). Operating cycle is time period in which parking at loading area is permitted.

Definition V.2 (Historical availability profile (HAP)). Historical availability profile of a loading area $d_{i}$ consists of the mean of the area's parking availability as a function of time,

$$
\hat{h}\left(d_{i}, t_{j}\right)=\frac{\sum_{k=1}^{m} \hat{a}_{k}\left(d_{i}, t_{j}\right)}{m}
$$



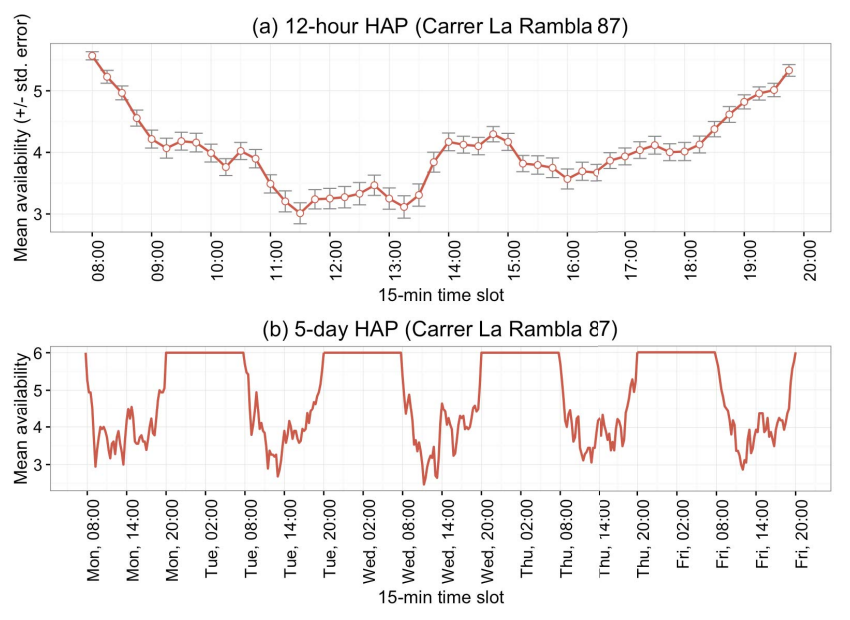

Fig. 6. 12-hour (a) and 5-day (b) HAPs for example loading area located in La Rambla street

where $m$ is the number of operating cycles for which parking availability is collected.

Since time is discrete we need to compute the parking availability for each time point of the operating cycle. However, in order to capture the previously discovered periodicities we use 15-minute discretisation bin. To put it differently, we first sample the time-stamped check-in data by accumulating the number of checked in vehicles over 15-minute sampling period. Thereafter, we compute the parking availability using (7) for each 15-minute time slot. Finally, HAPs are obtained by averaging computed availabilities using (8). For example, in Figure 6a a 12-hour HAP for previously observed loading area in La Rambla street is obtained by computing the average availability for each of the 15-minute time slot within 12hour operating cycle (from 08:00 to 20:00) over the 20-week monitoring period. Similarly, in Figure $6 \mathrm{~b}$ we obtained a 5day (weekly) HAP for the same area and operating cycle by computing averages for each of the 384 time slots in 5-day window. The computed availability patterns depend significantly on the area's location and the surrounding urban environment. For example, the observed area in La Rambla street is located next to the famous market place La Boqueria, one of the Barcelona's foremost tourist landmarks. The market has a very diverse selection of goods, mostly fresh food that needs to be replenished very frequently to avoid out-of-stock position. The 12-hour HAP plotted in Figure 6 a reflects the high turnover of deliveries throughout a whole day. However, notice that the mornings and evenings are less busy due to the lower trading volumes and, consequently, lower replenishment needs. Further, the popular visit times (12:00 - 14:00 and 16:00 - 19:00) are reflected in the plot as the busiest delivery periods. The subtle availability increase in the short period between 14:00 and 16:00 most likely reflects the lunch break and end of the first delivery shift. As we will see later in this section, this increase is common for all loading areas and can be observed more clearly in the global HAP aggregated for all
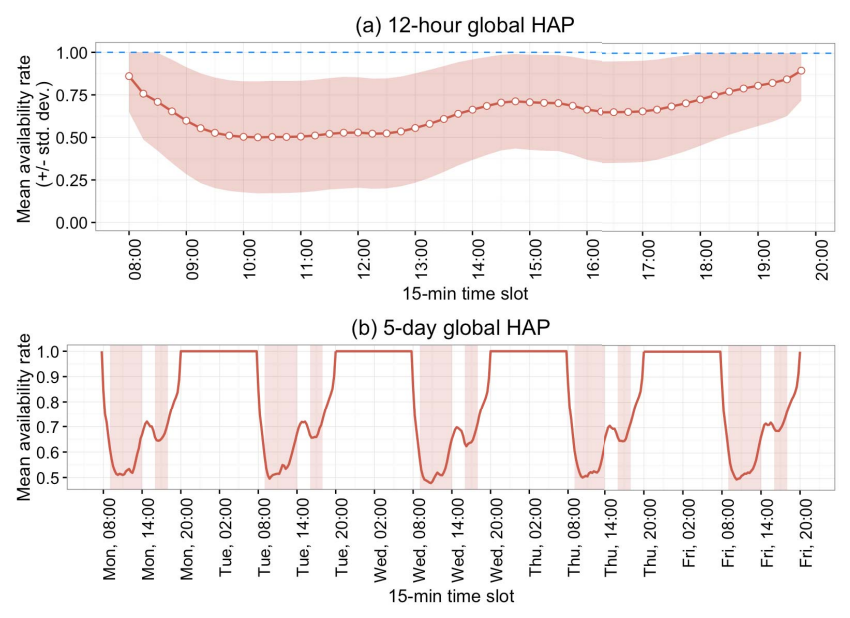

Fig. 7. 12-hour (a) and 5-day (b) global HAPs

loading areas. The weekly HAP plotted in Figure $6 \mathrm{~b}$ reveals very similar usage trends across all weekdays. As we expected, morning hours on Mondays are slightly busier than on the rest of the days due to the regular weekly stock replenishments.

As we previously noted, the usage trends depend on the loading areas, and thus we can observe different patterns in the different areas. However, instead of analysing each of the loading areas individually, we will take a look at the aggregated HAP computed by averaging the individual HAPs across all 1,990 loading areas. Such global view on availability fluctuations will give us a better understanding of the global mobility and delivery trends in the city of Barcelona. However, we noticed from the dataset that the parking availability at the particular loading area is closely correlated to the total number of authorized parking spaces, i.e. capacity of the area (plot is omitted to conserve space). Therefore, instead of aggregating previously computed availabilities, we also take in consideration the capacity of each of the areas and compute relative availabilities, i.e. $\hat{a}\left(d_{i}, t_{j}\right) / c_{d_{i}}$. In Figure 7 we plot the aggregated, i.e. global, 12-hour and 5-day HAPs. Recall from Figure 2 that there a large number of less popular loading areas with only couple of check-ins per day which explains high availability percentages in the plot. Nevertheless, the plot reveals two repeating valleys between 09:00 and 14:00, and 16:00 and 19:00, which corresponds to the first and second (i.e. morning and afternoon) delivery shift. As expected, the second delivery shift is significantly shorter and less busy. Also notice that the second shift on Friday is less active than on the other weekdays, most likely due to reduced working hours in many workplaces in Barcelona. The shift change period and late lunch break can be now observed more clearly as a sudden $30 \%$ availability increase between 14:00 and 16:00. Also, notice that all of the weekdays have very similar usage patterns. However, in Figure 7a relatively large standard deviation (red area) indicates instability of the observed HAPs. To put it differently, the individual HAPs significantly differ one from another, especially in busy time intervals. 


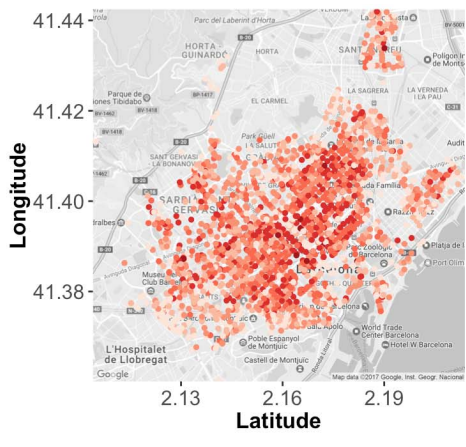

\section{HAP clustering}

The geographic layout of the city of Barcelona is distinguished by its balanced mixture of residential, commercial and touristic areas, and as such has a very strong influence on the delivery operations and the underlying mobility patterns. In order to understand them better, we group areas with similar HAPs and then analyse the geographic layout of the obtained clusters. We again normalise the individual HAPs with respect to areas' capacities (recall that we did the same in Section V-B), and thereafter apply average-linkage hierarchical clustering algorithm to partition loading areas based on their daily usage trends. The algorithm starts with 1,990 clusters, one for each area. The clusters are then sequentially combined into larger clusters until all loading areas end up being in the same cluster. At each step, the two clusters separated by the shortest distance are combined. The cluster-to-cluster distance is computed as the average pairwise Euclidean distance between 12-hour HAPs belonging to the areas in two different clusters. Finally, the result of the clustering is illustrated in Figure 8 . We cut the obtained dendrogram into 6 clusters. The very small standard deviations of per-cluster aggregated HAPs indicate high intra-cluster similarities. On the other hand, the aggregated HAPs exhibit very similar shapes with two valley in morning and afternoon, however with the different magnitudes. Therefore, the clusters mainly differ from each other by the usage intensity, i.e. the magnitude of the availability rate. Consequently, we order the clusters based on the average availability rate, i.e. from the least to the most occupied. The first cluster with 363 loading areas is the least popular one. This corresponds to the plot in Figure 1 where we noticed the large number of less popular areas with only couple of checkins per day. Similarly, the sixth cluster is the smallest, but the most popular one. It contains 16 most occupied loading areas. In order to detect the busiest delivery areas in the city (hotspots), in Figure 8 (right) we plotted location of each area on the map of Barcelona. Additionally, we coloured the markers based on their belonging clusters. The most popular areas (coloured in darker reds) are located in the central and the most developed part of the city. It is also interesting to notice that most of these popular areas are spread across only two districts - Ciutat Vella and Eixample, the most touristic and developed one. On the other hand, the less busy loading areas are located in the peripheral part of the city.

\section{AVAILABILITY PREDICTION}

We focus next on the short-term prediction of loading area availability. In particular, we are interested in predicting the total number of available parking spaces in the next time slot, i.e. 15-30 minutes ahead of time. This setup corresponds to most of the delivery scenarios in Barcelona where parking is planned on the fly, while all of the areas are reachable within 30 minutes from any part of the city. Additionally, for most of the loading areas maximum allowed parking time is 30 minutes. The benefits of the availability prediction are threefold: (1) it allows more accurate load balancing of loading areas, (2) it assists city governments in mobility management by providing information about expected delivery activity, and (3) it opens up new possibilities for mobile services such as delivery route planning. We compare several simple prediction models, and establish them as baselines with which other (more complex) models can be compared. We then present a more advanced prediction techniques that combine both historical and online availability data.

We denote by $x\left(d_{i}, t_{j}\right)$ a random variable representing the true parking availability at loading area $d_{i}$ at 15-minute time slot $t_{j}$ of an operating cycle. Similarly, we denote by $\hat{x}\left(d_{i}, t_{j}\right)$ the predicted parking availability for time slot $t_{j}$ of the same operating cycle.

\section{A. Historical model}

Historical prediction model (HM) $\hat{x}_{H M}\left(d_{i}, t_{j}\right)$ dynamically constructs a HAP based on all observations until the current time slot $t_{j}-1$, and returns the value at the corresponding HAP's time slot $t_{j}$. More formally,

$$
\hat{x}_{H M}\left(d_{i}, t_{j}\right)=\hat{h}\left(d_{i}, t_{j}\right)
$$

\section{B. Last value model (LV)}

Last value prediction model $(\mathrm{LV}) \hat{x}_{L V}\left(d_{i}, t_{j}\right)$ predicts that the availability at the current time slot $t_{j}-1$ remains constant as its last measured value $\hat{a}\left(d_{i}, t_{j}-1\right)$ for the next time slot $t_{j}$, i.e.

$$
\hat{x}_{L V}\left(d_{i}, t_{j}\right)=\hat{a}\left(d_{i}, t_{j}-1\right)
$$




\section{Linear regression model (LR)}

This model uses a mixture of the online data we acquire from the application and past averages of the availability data to train a linear regression model. The model formed for estimation of availability at area $d_{i}$ and timeslot $t_{j}$, $x_{L R}\left(d_{i}, t_{j}\right)$, is as follows

$$
\hat{x}_{L R}\left(d_{i}, t_{j}\right)=\beta_{1} \hat{a}\left(d_{i}, t_{j-1}\right)+\beta_{2} \hat{h}\left(d_{i}, t_{j}\right)+\gamma
$$

This model incorporates both the historical model for the specific area and the current availability patterns for that specific day to better estimate the current availability.

The parameters are estimated for each parking area and for each time slot using the previous data for the particular day. The following matrix denotes the training matrix for LR model for parking area :

$$
X_{d_{i}, t_{j}-1}=\left[\begin{array}{cc}
\hat{a}\left(d_{i}, t_{1}\right) & \hat{h}\left(d_{i}, t_{2}\right) \\
\hat{a}\left(d_{i}, t_{2}\right) & \hat{h}\left(d_{i}, t_{3}\right) \\
\vdots & \vdots \\
\hat{a}\left(d_{i}, t_{j-2}\right) & \hat{h}\left(d_{i}, t_{j-1}\right)
\end{array}\right]
$$

Corresponsing ground truth vector for training is as follows:

$$
Y_{d_{i}, t_{j-1}}=\left[\begin{array}{c}
a\left(d_{i}, t_{2}\right) \\
a\left(d_{i}, t_{3}\right) \\
\vdots \\
a\left(d_{i}, t_{j-1}\right)
\end{array}\right]
$$

where $t_{1}$ time slot value corresponds to first time slot of the operating cycle. We can find the parameters of the model using regular solutions to linear regression problems such as leastsquares estimation.

Intercept value $(\gamma)$ is also added to the model but from the results of our data we have seen that a model without intercept seems to decrease the estimation error.

One also notes that we have scarce data to estimate from when $t_{j}$ is small, i.e. at the beginning of operating cycle. This scarcity can cause unstable and unreliable results in the estimation phase. To tackle this issue we have used the p-value of the parameters to measure the significance of the relations of the variables. We have chosen $p=0.05$ as a threshold for significance which is the value used generally in the literature. According to the result of estimation if calculated significance is less than the threshold we use the the historical model estimate, $\hat{x}_{H M}\left(d_{i}, t_{j}\right)$, instead. In summary:

$$
\hat{x}_{L R}\left(d_{i}, t_{j}\right)= \begin{cases}\hat{x}_{L R}\left(d_{i}, t_{j}\right), & \text { if } p<=0.05 \\ \hat{x}\left(d_{i}, t_{j}\right), & \text { otherwise }\end{cases}
$$

\section{Linear regression model with aggregated HAPs (LR-A)}

Recall from Section III that for some of the loading areas we have very few observed check-ins. The availability of these areas is harder to predict with the proposed models due to sparseness of the observations. On the other hand, in Section V-C we saw that loading areas can be efficiently clustered using normalised HAPs, and thus less sparse aggregated HAP can be obtained for each cluster. Therefore, in order to overcome data sparsity issues, we propose to extend previously defined LR model by aggregating historical availability information. To put it differently, instead of using a single HAP of the specific area, we cluster areas with similar HAPs and compute aggregated representative HAP. Forecasts are then performed by applying LR model with the corresponding representative HAP to each loading area in the cluster, individually. As we will see in the next section, in certain cases these grouped models can avoid overfits, reduce the impact of outliers, and successfully capture common trends.

We compute cluster representative HAP by averaging historical availabilities across all loading areas contained in the corresponding cluster, i.e.

$$
\hat{h}\left(C_{k}, t_{j}\right)=\frac{1}{\left|C_{k}\right|} \sum_{d_{i} \in C_{k}} \hat{h}\left(d_{i}, t_{j}\right)
$$

where $C_{k}$ stands for arbitrary cluster. The ground truth vector $Y_{d_{i}, t_{j-1}}$ remains the same, while the training matrix $X_{d_{i}, t_{j}-1}$ is updated as follows

$$
X_{d_{i}, t_{j}-1}=\left[\begin{array}{cc}
\hat{a}\left(d_{i}, t_{1}\right) & \hat{h}\left(C_{k}, t_{2}\right) \\
\hat{a}\left(d_{i}, t_{2}\right) & \hat{h}\left(C_{k}, t_{3}\right) \\
\vdots & \vdots \\
\hat{a}\left(d_{i}, t_{j-2}\right) & \hat{h}\left(C_{k}, t_{j-1}\right)
\end{array}\right], \forall d_{i} \in C_{k}
$$

We experimented with different clustering algorithms and similarity measures, and we found simple k-nearest neighbour method $(\mathrm{kNN})$ and cosine similarity to give accurate results. Therefore, we compute the similarity between areas as follows

$$
\operatorname{sim}\left(d_{i}, d_{j}\right)=\frac{\sum_{k=1}^{48} \hat{h}\left(d_{i}, t_{k}\right) \hat{h}\left(d_{j}, t_{k}\right)}{\sqrt{\sum_{k=1}^{48} \hat{h}\left(d_{i}, t_{k}\right)^{2}} \sqrt{\sum_{k=1}^{48} \hat{h}\left(d_{j}, t_{k}\right)^{2}}}
$$

\section{EXPERIMENTAL EVALUATION}

\section{A. Experimental setup}

Our simulations and experiments are based on real-world check-in data introduced in Section III. In order to simulate ground truth data we assume $100 \%$ adoption rate and estimate the true availabilities using (7), i.e.

$$
x\left(d_{i}, t_{j}\right)=c_{d_{i}}-1.368 o_{c}^{+}\left(d_{i}, t_{j}\right)
$$

However, we also generate the input data stream by sampling the original data with respect to different adoption rates $p$. In other words, each check-in from the original dataset is randomly selected in the input stream with the probability $p$. We use the generated input stream to predict the true availability.

In order to estimate how accurately the prediction models will perform in practice, we use time series cross-validation strategy known as rolling forecasting origin [5]. With a rolling forecasting origin, we create an initial window that contains the first $k$ data points as the first training set to estimate the prediction model. We compute the error on the forecast for the next data point $k+1$, and then shift the window by one data point. We repeat the procedure until no data point left. In our case, we use $k=3360$ data points, i.e. time slots, as 


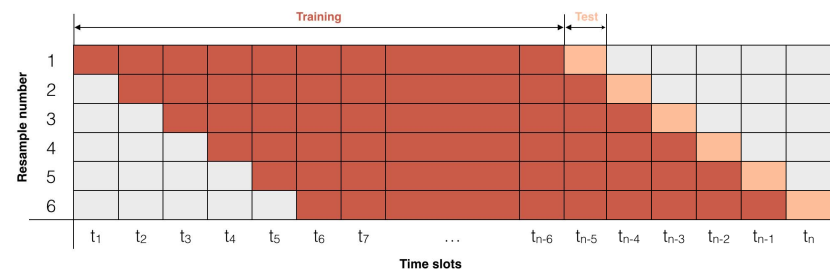

Fig. 9. Example of rolling forecasting origin strategy

training set window. This corresponds to 70 operating cycles and $80 \%$ of the whole dataset. In Figure 9 we further illustrate the employed evaluation strategy.

Finally, we consider the mean squared error (MSE) between the estimated and true availability as a performance measure for the proposed models, i.e.

$$
M S E\left(d_{i}\right)=\frac{1}{n-k-1} \sum_{j=k+1}^{n}\left(\hat{x}\left(d_{i}, t_{j}\right)-x\left(d_{i}, t_{j}\right)\right)^{2}
$$

We have also considered looking at vacant parking space existence; a boolean variable checking if the model has correctly identified the presence of at least one available parking space. However, since users can make more confident decisions to use a parking place based on the number of vacant places (a higher number of vacant space increases the probability of at least one vacant space by the time user has arrived at the specific area) we have opted to use the mean square error instead. Our methods have shown very high accuracy levels in terms of estimation of vacant space existence and estimation of exact vacant space size is more challenging when these two performance criteria are compared.

\section{B. Results}

We evaluate the performance of the proposed predictive models on five input streams sampled from the original check-in dataset. The sampling was conducted using previously described simple random method with $p \in$ $\{0.5,0.6,0.7,0.8,0.9\}$. This way, we simulate different adoption stages of the new system in order to assess how the adoption rate reflects on the performance of the models under evaluation.

Figure 10 shows the MSE performance of the models for different adoption rates. Here, the total MSE is aggregated over all time slots in the test dataset. As expected, for all

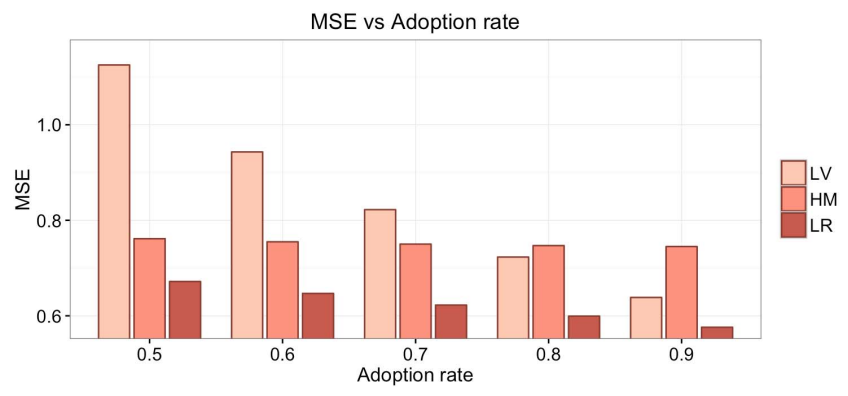

Fig. 10. MSE performance for different adoption rates models, the MSE decreases as the adoption rate increases. In other words, the models perform better on less sparse input streams. However, the performance increase is not the same for all models. Notice that the MSE for the LV drops from 1.125 for $p=0.5$ to 0.638 for $p=0.9$ (43.29\% accuracy increase). On the other hand, the performance increase for the HM is less significant, i.e. the MSE drops from 0.761 for $p=0.5$ to 0.745 for $p=0.9$ (2.10\% accuracy increase). Also notice that the LV outperforms the HM for $p=0.8$ and $p=0.9$. The LV relies on the estimation of the current availability whose accuracy strongly depends on the system's adoption rate, i.e. sparsity of input stream. This is not the case with the HM where the estimated availabilities are aggregated for each time slot of the operating cycle over the entire input stream. Finally, from the plot it can be observed that the LR significantly outperforms the other models for all input streams. In other words, the LR is able to successfully combine online and historical prediction approach, and outperform them both when applied separately. Similar to the LV and HM, the accuracy of the LR increases with the adoption rate, i.e. the MSE drops from 0.672 for $p=0.5$ to 0.576 for $p=0.9$ ( $14.29 \%$ accuracy increase).

In addition to the total MSE, we also compute the MSE for each of the 48 time slots of the operating cycle and study how the prediction models perform with respect to different times of day. In Figure 11 we plot the MSE per time slot for different adoption rates $p$. All of the plots exhibit two distinct peaks, of which the higher (left-hand) peak occurs between time slot 5 and 25 (09:00 - 14:00) which corresponds to morning delivery shift, and the lower (right-hand) peak occurs between time slot 33 and 45 (16:00 - 19:00) which corresponds to afternoon delivery shift. Notice that these patterns are negatively correlated to the ones in Figure 7 where we plotted relative availabilities against different time slots of the operating cycle. This means that all of the models perform slightly worse during busy periods when check-in patterns are less stable. Since it is more difficult to find a parking space during busy periods, deliverers tend to go from one loading area to another in search for a parking space. Obviously, this makes deliveries to some extent disorganized and thus harder to predict.

Figure 11 also shows that at most times of day the LR outperforms both LV and HM. However, the exception to this can be observed at the beginning of the operating cycle (time slots 1-10), i.e. in morning hours (08:00 - 10:00), for all adoption rates $p$. The reason for this lies in the fact that the LR relies on current trends which can be difficult to infer at the start of a day when we have only few data points at disposal. Recall from Section VI-C that we tackle this scarcity problem by using p-values for the model parameters to test the significance of the relation between the variables (i.e. online and historical availability). If the p-value falls above 0.05 threshold, instead of using the trained LR model for prediction, we use the HM model. As shown in Figure 12, with this simple trick we managed to significantly improve the initial performance of the LR model at the beginning of the operating cycle. 


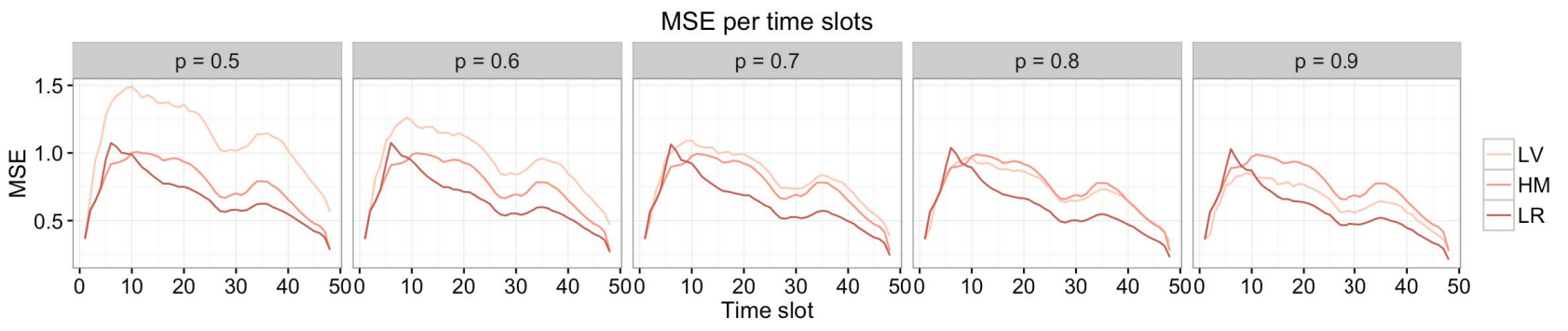

Fig. 11. MSE performance per time slots

As we discussed in the previous section, the performance of the LR model can be further improved in certain cases by grouping similar loading areas and computing aggregated HAP (LR-A model). Recall from Section III that check-ins are unequally distributed among loading areas, and thus for some of the less popular areas in city's periphery, the collected data can be sparse and bursty. In these special cases, clustering and aggregating arise as a means to reduce the noise and avoid overfitting. We will demonstrate the performance of the LR-A model on an isolated case depicted with Figure 13.

In Figure 13a we plot individual HAPs of 4 similar loading areas grouped together using k-nearest neighbour $(\mathrm{kNN})$ method. Interestingly, the typical 2-valley shape is harder to observe in these unsmooth plots. This suggests that data recorded at these specific areas is quite noisy and bursty. Additionally, we notice that the average number of check-ins per day for the grouped areas is only 10.13. Therefore, instead of building 4 individual models that might be vulnerable to overfitting, we compute aggregated HAP and use it to build a single, but more robust, prediction model. In Figure $13 \mathrm{~b}$ we compare the performance of individual LR models and aggregated LR-A model. The plot shows that LR-A outperforms LR for all of the grouped areas. More precisely, we reduce the MSE for $7.52 \%$ for area $8656,6.65 \%$ for area 9463, $12.32 \%$ for area 9987 , and $11.46 \%$ for area 10527 .

\section{RELATED WORK}

In recent years, the mobility patterns in urban freight networks have attracted a certain amount of attention in the research community (e.g. [6]-[9]). However, obtaining data which would allow a large scale study is often very difficult (or impossible), mostly due to privacy issues. Therefore, many studies relies on information gathered from surveys carried out

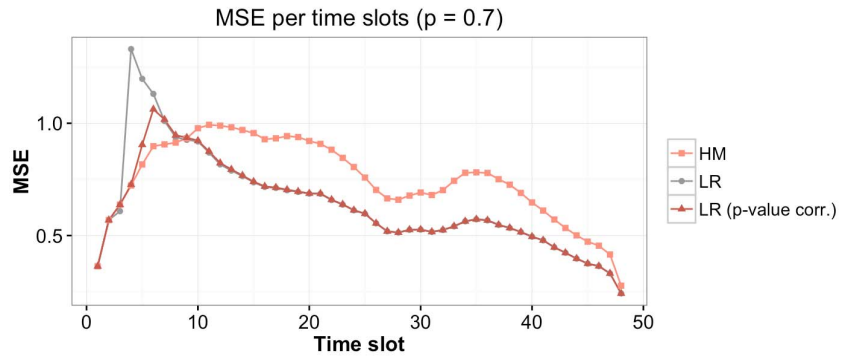

Fig. 12. MSE performance of the initial and adjusted LR model among freight distributors (e.g. [10], [11]). In our paper, we use real-life check-in dataset collected from the novel parking regulation system based on mobile technologies.

We use historical availability profiles (HAPs) of the loading areas in order to understand daily and weekly delivery trends. The similar concepts can be often found in research works that focus on analysis of human mobility patterns. For example, Froehlich et al. [12] employ the concept of DayView to study bicycle station usage from Barcelona's shared bicycling system. A DayView is calculated by averaging station data that matches certain criteria into a 24 hour window, discretised into five-minute bins (288 bins/day). Additionally, in order to investigate how usage patterns are shared across stations and geographically distributed in the city, Froehlich et al. cluster stations based on their DayViews. They show that the spatial layout of a city has a strong influence on the movement patterns and social behaviours. A similar research has been conducted in [13], where the DayViews are replaced with local ActivityCycles computed for each station and day of a week. Again, in order to get a spatial picture of the mobility pattern in the city, these local ActivityCycles are paired with the stations geo-coordinates and visualised on a heat map.

In [14] Xu et al. introduce an algorithm to construct the historical availability profiles (HAP) by computing estimated values for the mean and the variance of true parking availability for an arbitrary street block. The algorithm relies on PhonePark system which is able to automatically detect parking events using drivers' mobile phones equipped with GPS and/or accelerometer. Here, detected parking events correspond to check-ins in our case. Additionally, since not all drivers are equipped with PhonePark system, its market penetration ratio is also considered in [14] (i.e. share of drivers with PhonePark
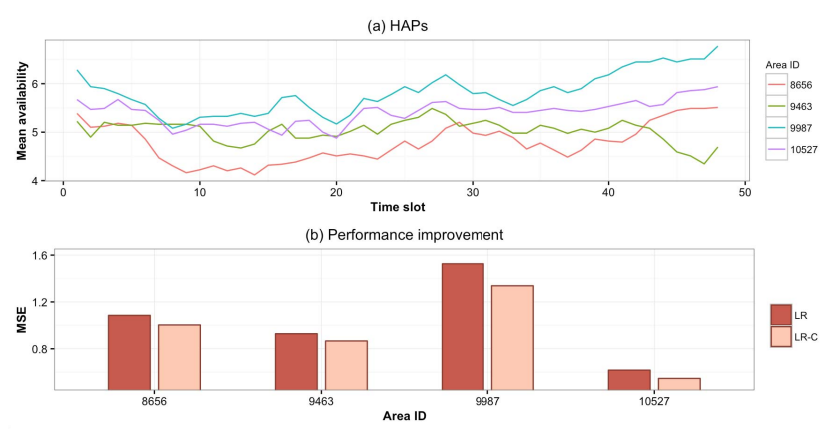

Fig. 13. MSE performance of LR and LR-A model 
enabled phones). Similarly, we use adoption rate in our work to simulate different adoption stages of the new system. Finally, while $\mathrm{Xu}$ et al. estimate the current availability of street blocks, we focus on short-term availability prediction of loading areas using obtained historical and current trends.

Several recent studies have investigated the problem of predicting parking availability. In [15] and [16], a continuoustime Markov model is built to predict the parking availability in ad hoc networks. In [17], a recurrent neural network is employed to forecast the parking occupancy using data collected from a wide network of parking sensors installed on-street in the city of Santander, Spain. Similarly, Zheng et al. [18] analyse the performance of different machine learning methods (neural network, regression tree, support vector regression) in predicting parking availability from the real-time car parking information that has been collected and disseminated by the City of San Francisco, USA and the City of Melbourne, Australia. Additionally, they consider several feature sets consist of past observations, time of day and day of week. In [19], Chen et al. propose Generalized Additive Models for availability prediction of shared bike and car parking lots in the city of Dublin, Ireland. They consider even more features such as time of day, time of year, day type, weather, temperature, humidity and past occupancy observations. Some other studies (e.g. [13], [20]) view the parking data as a one-dimensional time series and explore the use of time series models such as Autoregressive Integrated Moving Average (ARIMA).

\section{CONCLUSION}

We first presented the characteristics of data collected by a new parking system for urban freight deliverers which has been released as a part of Barcelona's sustainable urban mobility plan. The system allows deliverers to check-in at the designated loading areas using a mobile application, and thus provide real-time occupancy information. The mobile application eliminates the need for hardware based investments or technologies such as sensor infrastructures for parking lots, and provides more information about occupancy such as the licence of the trucks in the parking area. We then presented an exploratory analysis of the dataset in order to detect temporal patterns in urban freight transport. Due to phased adoption of the system and illegal parking practices, we had to collect additional occupancy information from the field to estimate the real availability of loading areas. We build individual historical availability profiles (HAPs) for each parking area. We showed that HAPs can provide valuable information about delivery trends and can be efficiently used for detecting hotspots and rush hours, as well as forecasting the future availability for any specific area.

We successfully combined online and historical prediction approach using simple linear regression which can outperform them both when applied separately. In other words, the proposed model incorporates both the historical model (i.e. HAP) for the specific loading area and the current availability patterns for that specific day to better estimate the current availability. The estimated availability values are considered accurate enough to efficiently utilize a parking area recommendation system in Barcelona according to the city government. Parking availability forecasting opens up new possibilities for delivery route planning and better managing of freight transport infrastructures. The proposed prediction models can be used for route planning and itinerary generation for more effective urban freight transportation.

\section{REFERENCES}

[1] U. Nations, World Urbanization Prospects 2014: Highlights. United Nations Publications, 2014.

[2] European Commission, Urban Freight Transport and Logistics: An Overview of the European Research and Policy. European Commission Publications, 2006.

[3] CIVITAS Initiative WIKI consortium, Smart choices for cities: Making urban freight logistics more sustainable, 2015.

[4] C. Jaffeux and P. Wieser, Essentials of logistics and management. CRC Press, 2012.

[5] R. J. Hyndman and G. Athanasopoulos, Forecasting: principles and practice. OTexts, 2014.

[6] P. Pluvinet, J. Gonzalez-Feliu, and C. Ambrosini, "Gps data analysis for understanding urban goods movement," Procedia-Social and Behavioral Sciences, vol. 39, pp. 450-462, 2012.

[7] X. Yang, Z. Sun, X. Ban, and J. Holguín-Veras, "Urban freight delivery stop identification with gps data," Transportation Research Record: Journal of the Transportation Research Board, no. 2411, pp. 55-61, 2014.

[8] M. Tozzi, M. V. Corazza, and A. Musso, "Recurring patterns of commercial vehicles movements in urban areas: the parma case study," Procedia-Social and Behavioral Sciences, vol. 87, pp. 306-320, 2013.

[9] J. Muñuzuri, P. Cortés, L. Onieva, and J. Guadix, "Modelling peak-hour urban freight movements with limited data availability," Computers \& Industrial Engineering, vol. 59, no. 1, pp. 34-44, 2010.

[10] - "Estimation of daily vehicle flows for urban freight deliveries," Journal of Urban Planning and Development, vol. 138, no. 1, pp. 43-52, 2011.

[11] T. Cherrett, J. Allen, F. McLeod, S. Maynard, A. Hickford, and M. Browne, "Understanding urban freight activity-key issues for freight planning,” Journal of Transport Geography, vol. 24, pp. 22-32, 2012.

[12] J. Froehlich, J. Neumann, and N. Oliver, "Sensing and predicting the pulse of the city through shared bicycling." in IJCAI, vol. 9, 2009, pp. $1420-1426$.

[13] A. Kaltenbrunner, R. Meza, J. Grivolla, J. Codina, and R. Banchs, "Urban cycles and mobility patterns: Exploring and predicting trends in a bicycle-based public transport system," Pervasive and Mobile Computing, vol. 6, no. 4, pp. 455-466, 2010.

[14] B. Xu, O. Wolfson, J. Yang, L. Stenneth, S. Y. Philip, and P. C. Nelson, "Real-time street parking availability estimation," in 2013 IEEE 14th International Conference on Mobile Data Management, vol. 1. IEEE, 2013, pp. 16-25.

[15] M. Caliskan, A. Barthels, B. Scheuermann, and M. Mauve, "Predicting parking lot occupancy in vehicular ad hoc networks," in 2007 IEEE 65th Vehicular Technology Conference-VTC2007-Spring. IEEE, 2007, pp. 277-281.

[16] A. Klappenecker, H. Lee, and J. L. Welch, "Finding available parking spaces made easy," Ad Hoc Networks, vol. 12, pp. 243-249, 2014.

[17] E. I. Vlahogianni, K. Kepaptsoglou, V. Tsetsos, and M. G. Karlaftis, "A real-time parking prediction system for smart cities," Journal of Intelligent Transportation Systems, vol. 20, no. 2, pp. 192-204, 2016.

[18] Y. Zheng, S. Rajasegarar, and C. Leckie, "Parking availability prediction for sensor-enabled car parks in smart cities," in Intelligent Sensors, Sensor Networks and Information Processing (ISSNIP), 2015 IEEE Tenth International Conference on. IEEE, 2015, pp. 1-6.

[19] B. Chen, F. Pinelli, M. Sinn, A. Botea, and F. Calabrese, "Uncertainty in urban mobility: Predicting waiting times for shared bicycles and parking lots," in 16th International IEEE Conference on Intelligent Transportation Systems (ITSC 2013). IEEE, 2013, pp. 53-58.

[20] F. Yu, J. Guo, X. Zhu, and G. Shi, "Real time prediction of unoccupied parking space using time series model," in Transportation Information and Safety (ICTIS), 2015 International Conference on. IEEE, 2015, pp. 370-374. 